Association for Information Systems AIS Electronic Library (AISeL)

Wirtschaftsinformatik Proceedings 2005

Wirtschaftsinformatik

February 2005

\title{
The Impact of Semantic Web Technologies on Job Recruitment Processes
}

\author{
Christian Bizer \\ Freie Universität Berlin \\ Ralf Heese \\ Humboldt-Universität zu Berlin \\ Malgorzata Mochol \\ Freie Universität Berlin \\ Radoslaw Oldakowski \\ Freie Universität Berlin \\ Robert Tolksdorf \\ Freie Universität Berlin \\ See next page for additional authors
}

Follow this and additional works at: http://aisel.aisnet.org/wi2005

\section{Recommended Citation}

Bizer, Christian; Heese, Ralf; Mochol, Malgorzata; Oldakowski, Radoslaw; Tolksdorf, Robert; and Eckstein, Rainer, "The Impact of Semantic Web Technologies on Job Recruitment Processes" (2005). Wirtschaftsinformatik Proceedings 2005. 72.

http://aisel.aisnet.org/wi2005/72 
Authors

Christian Bizer, Ralf Heese, Malgorzata Mochol, Radoslaw Oldakowski, Robert Tolksdorf, and Rainer Eckstein 
In: Ferstl, Otto K, u.a. (Hg) 2005. Wirtschaftsinformatik 2005: eEconomy, eGovernment, eSociety; 7. Internationale Tagung Wirtschaftsinformatik 2005. Heidelberg: Physica-Verlag

ISBN: 3-7908-1574-8

(C) Physica-Verlag Heidelberg 2005 


\section{The Impact of Semantic Web Technologies on Job Recruitment Processes}

\section{Christian Bizer}

Freie Universität Berlin

\section{Ralf Heese}

Humboldt-Universität zu Berlin

\section{Malgorzata Mochol, Radoslaw Oldakowski, Robert Tolksdorf}

Freie Universität Berlin

\section{Rainer Eckstein}

Humboldt-Universität zu Berlin

Abstract: Using Semantic Web technologies in the domain of online recruitment could substantially increase market transparency, lower the transaction costs for employers, and change the business models of the intermediaries involved. In this paper, we describe how online recruitment processes can be streamlined using Semantic Web technologies. We analyze the realization chances of such a scenario from the technological and business viewpoint. We describe a prototypical implementation of the required technological infrastructure and analyze the potential economic impacts, the interests of the involved parties, and potential changes to their business models.

Keywords: Semantic Web, Recruitment Process, Electronic Market, Semantic Matching

\section{Introduction}

The Internet has already evolved into the primary medium for recruitment and employment processes. Over $53 \%$ of recently filled positions in Germany are the result of an online job posting and according to most forecasts this share is likely to increase further [WiMo03].

Despite these achievements, the information flow in the online labour market is far from optimal. A large number of online job portals have sprung up, dividing the online labour market into information islands and making it close to impossi- 
ble for a job seeker to get an overview of all relevant open positions. Their strong market position, as the prime starting point for job seekers, allows job portals to charge employers high fees for publishing open positions. Due to these costs employers publish their job postings only on a small number of portals, which prevents the offers from reaching all qualified applicants.

Employers often receive a large number of applications for an open position, due to the strained situation of the labour market. The costs of manually preselecting potential candidates have risen and employers are searching for means to automate the preselection of candidates.

Thus, employers would like to decrease transaction costs for publishing job postings and for preselecting appropriate candidates. Job seekers would profit from increased transparency in the labour market in their search for a matching position. In this paper, we argue that both goals could simultaneously be reached by using Semantic Web technologies within recruitment processes.

The basic idea of the Semantic Web [BeHe01, HeBe02] is to extend the Web, in addition to classic HTML pages, with machine-understandable data. The objective is to use the Web like a global distributed knowledge store which can be leveraged by applications to perform tasks automatically. The base technologies for realizing this vision are:

- Uniform Resource Identifiers (URIs) as a global identification mechanism for resources and terms used to describe them,

- the Resource Description Framework (RDF) as basic data model together with its XML-based serialisation syntax for publishing data on the Web [W3C04a],

- the Web Ontology Language (OWL) extending RDF with terms and concepts for expressive knowledge representation [W3C04c].

The development of the Semantic Web is a joined effort of scientific (MIT, Stanford, ILRT etc.) and business institutions (HP, IBM, Nokia etc.) led by the World Wide Web Consortium (W3C). There are substantial research and development investments in semantic technologies by the mayor players of the IT market [QuKa04]. The first large scale prototypes have been deployed in the domains of biomedical and bibliographic data integration [Schr04, QuKa04].

The technological foundations of the Semantic Web are getting mature. What is still unclear are the economic effects the new technology will have on markets and enterprises. With our research, we approach potential impacts of semantic technologies from the business and the technological viewpoint, in order to make predictions about the influence of the new technologies on markets, enterprises, and individuals. Who will benefit? Who will lose his market position or will have to change his business model? This analysis of the participants' roles combined with the analysis of technological restrictions allows us to project whether a scenario is likely to be realized. 
In section 2 of this paper, we describe how online recruitment processes can be streamlined using Semantic Web technologies. We describe our prototypical implementation of the required technological infrastructure in section 3, focusing on the ontologies used, the data integration infrastructure, and on semantic matching services. In section 4, we analyze the potential economic impacts of the realization of the scenario from the perspectives of the different participants.

\section{Supporting Recruitment Processes with Semantic Web Technologies}

A usual recruitment process seen from the organization's viewpoint can be divided into four phases:

1. Requirements analysis

2. Publishing the job posting

3. Receiving and preselecting applications

4. Final recruitment decision.

In this section, we describe how the different phases can be supported by Semantic Web technologies. The central building blocks of the scenario are the use of controlled vocabularies to annotate job postings and applications, and the use of RDF to include these descriptions into process documents. Figure 1 gives an overview of the process described below.

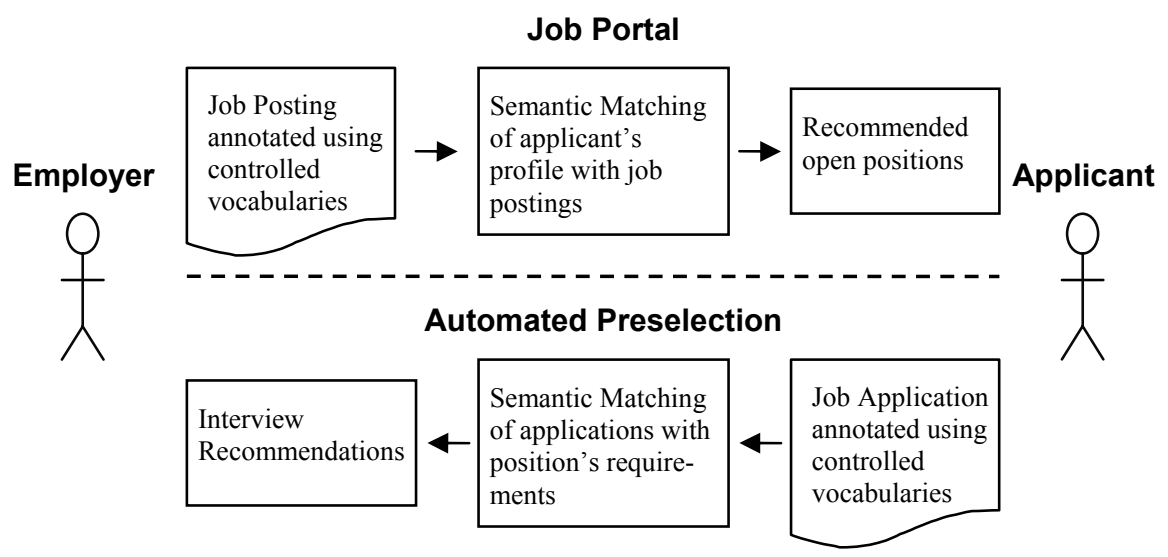

Figure 1: Recruitment process using Semantic Web technologies. 


\subsection{Requirements Analysis}

The requirements for an open position are usually conceived by the operating department together with the human resource department. Nowadays the resulting job posting describing the requirements for an open position is written in free text. The usage of free text limits the machine processability of postings in the later phases of the recruitment process. In contrast to a free text description, the usage of a common "language" in the form of a set of controlled vocabularies for describing the details of a job posting would facilitate communication between all parties involved and would open up the potential of the automation of various tasks within the process.

\subsection{Publication of the Job Posting}

There are a large number of commercial job portals, like Monster ${ }^{1}$, JobPilot ${ }^{2}$ or StepStone ${ }^{3}$, competing to publish job postings for a publication fee. The different portals represent various audiences and are specialized in certain geographic regions. As publication fees easily add up, employers publish their postings only on a limited number of portals. The second publication channel for job postings is the employer's own website. Publishing postings on the corporate website is cheap but reaches only a very limited audience, because the indexing capabilities of current search engines like Google are too imprecise to support directed searches for open positions.

In order to improve market transparency several public bodies like the German Federal Employment Office (BA) ${ }^{4}$ and the Swedish National Labour Market Administration $(\mathrm{AMS})^{5}$ have started projects to integrate open positions in a central database. In both projects, participating employers use terms from a controlled vocabulary to categorize their postings and send them to the central database using variations of the HR-XML ${ }^{6}$ data format. The collected postings are published through a central portal and are forwarded to commercially run job portals. The problem with these projects is that the whole market depends on one central database - an approach to which many market participants object and which can be very error-prone as experience from the German project shows [Cross03].

Using Semantic Web Technologies to distribute job postings would increase market transparency together with avoiding the bottleneck of a central database. Or-

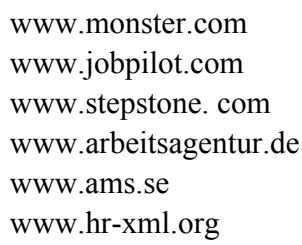


ganizations would publish annotated job postings directly on their corporate websites using terms from a set of controlled vocabularies and the RDF data format. The postings could be then crawled directly by all interested job portals, meaning that the centralised approach would be replaced with a distributed approach. Consequently, all job portals would operate on the same information and postings would reach more applicants, resulting in higher market transparency.

Another benefit from having postings annotated with terms from a controlled vocabulary is that the terms can be combined with background knowledge about an industrial domain. Job portals could offer semantic matching services which would calculate the semantic similarity between job postings and applicants' profiles based on background knowledge about how different terms are related. For example, if Java programming skills are required for a certain job and an applicant is experienced in Delphi, the matching algorithm would consider this person's profile a better match than someone else's who has the skill SQL, because Delphi and Java are closer related than SQL and Java. This approach allows the comparison of job position postings and applicants' profiles using background knowledge instead of merely relying on the containment of keywords, like traditional search engines do.

\subsection{Receiving and Preselecting Job Applications}

Employers often receive a large number of applications for an open position, due to the strained situation of the labour market. In order to reduce handling costs employers have begun to prefer online applications over classic paper applications. Other companies like $\mathrm{IBM}^{7}$ or Volkswagen ${ }^{8}$ have set up web forms for receiving applications, where applicants have to re-enter the basic facts of their application. This data is used for the automated preselection of candidates fulfilling the basic requirements and thus reducing preselection costs.

Both applicants and employers would benefit from the semantic annotation of job applications in addition to the classic free text application. Applicants could reuse their RDF profiles and send them to different employers instead of having to fill numerous web forms. Employers use the annotations for automating the preselection process by matching their minimal requirements with the applications.

Another issue of this phase is proofing testimonials of experience and education. Testimonials could be attached to applications in the form of RDF statements which are digitally signed by the issuing university or organization. The process of validating digitally signed testimonials can be automated, further reducing costs.

\footnotetext{
https://forms.bpfj.intronet.com/ibm/Forms/emea/expro/GeneralApp.jsp https:/www.vw-personal.de/content/www/de/bewerbung/onlinebewerbung.html
} 


\subsection{Interviews and Recruitment Decision}

Face-to-face contact with candidates is indispensable for the final recruitment decision. Thus, this phase of the recruitment process, where candidates are interviewed and where their soft and technical skills are evaluated through face-to-face meetings or even in assessment centres will still be done manually in the future.

\section{Technical Realization}

In this section we present our prototypical implementation of the technical infrastructure needed to realize the scenario described above. We focus on how vocabularies can be derived from standards already in use within the recruitment domain; on how the data integration infrastructure can be coupled with existing non-RDF human-resource systems, and on matching algorithms for calculating the similarity of job requirements and applicants' profiles. Our prototypical implementation is based on existing open source components which we have extended with scenario-specific features.

Our labour market scenario implies three basic roles:

- Information Providers who publish open positions and company background information using controlled vocabularies and the RDF data format.

- Information Aggregators who crawl the published information and present it to the information users in the desired format and level of aggregation. Combining job postings with background knowledge about the industrial sector allows the aggregators to offer semantic matching services. The usage of URIs as employer identification mechanism allows the enrichment of postings with information about employers from different external information services.

- Information Consumers who use one portal of their choice as central access point to all published open positions and background information about employers, instead of collecting information fragments from different sites.

Figure 2 illustrates the role model for the scenario and gives an overview of the technical architecture. 


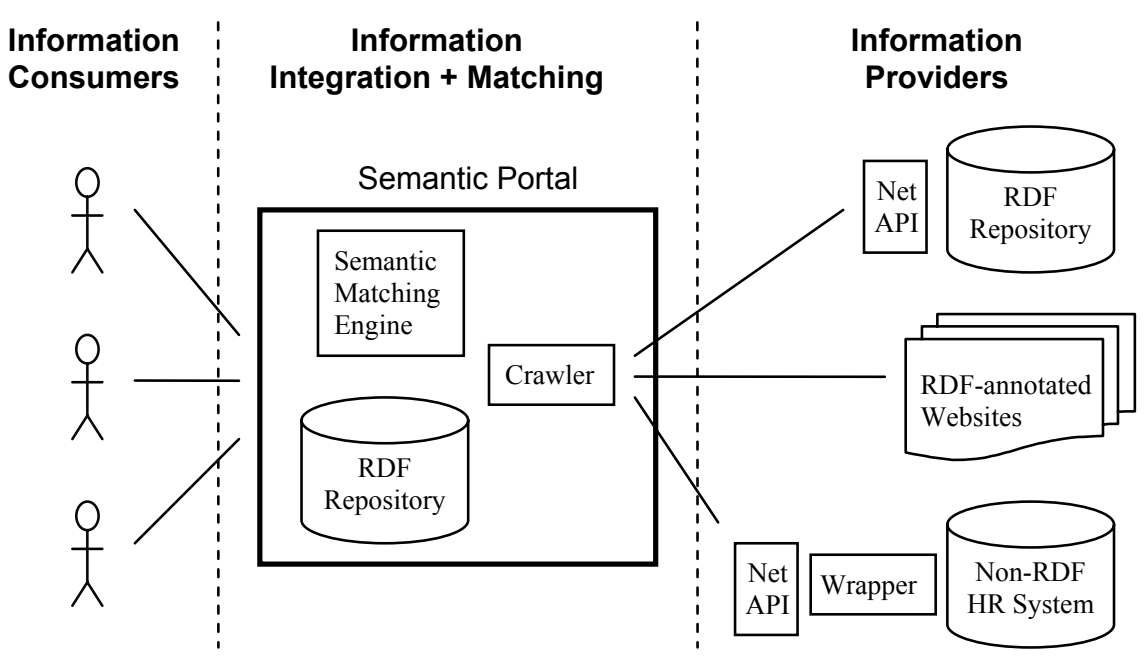

Figure 2: Scenario architecture and participants' roles.

\subsection{Ontologies}

The data exchange between employers and job portals is based on a set of vocabularies which provide shared terms for describing occupations, required skills and educational background. A wide range of standards for process documents and classification schemas has already been developed for recruitment. In the following, we give an overview about the relevant standards and describe how we integrated them into the ontology used by our prototype.

\subsubsection{Process Documents}

The most widely used standard for process documents like job postings and applications is the HR-XML standard. HR-XML has been agreed on by the HRConsortium $^{9}$, an independent, non-profit organization dedicated to the development and promotion of a standard suite of XML specifications to enable ebusiness and the automation of human resources-related data exchanges. HRXML is a library of more than 75 interdependent XML schemes. The XML schemes define the data elements for particular HR transactions, as well as options and constraints governing the use of these elements. The HR-XML Consortium has produced schemes covering major processes, as well as component schemes used across multiple business processes.

www.hr-xml.org 


\subsubsection{Taxonomies}

There are several taxonomies available for the description of skills, classification of job profiles and industrial sectors. These taxonomies provide the vocabulary used within process documents.

- Standard Occupational Classification System $(S O C)^{10}$ classifies workers into occupational categories (23 major groups, 96 minor groups, and 449 occupations).

- Occupation Code (Berufskennziffer $-B K Z)^{11}$ is a German version of SOC System. It classifies workers into 5597 occupational categories according to occupational definitions.

- North American Industry Classification System (NAICS) ${ }^{12}$ provides industry sector definitions for Canada, Mexico, and the United States to facilitate economic analyses.

- Classification of Industry Sector $(W Z 2003)^{13}$ is a German classification standard for economic activities.

\subsubsection{Our Human Resource Ontology}

We have created a human resource ontology (HR ontology) by integrating existing widespread used standards and classifications. The ontology is divided into subontologies (see Figure 3), which are used in both job posting and job application descriptions. We adapted the Job Position Seeker and Job Position Posting segments from HR-BA-XML (German version of HR-XML). To specify the industry sub-ontology we utilize both the German Classification of the Industry Sector (WZ2003) and the North American Industry Classification System (NAICS). As occupational classification we utilize the German Occupation Code (BKZ) and the Standard Occupational Classification (SOC) System.

\footnotetext{
http://www.bls.gov/soc

http://www.arbeitsamt.de/hst/markt/news/BKZ\alpha.txt

http://www.census.gov/epcd/www/naics.html

http://www.destatis.de/allg/d/klassif/wz2003.htm
} 


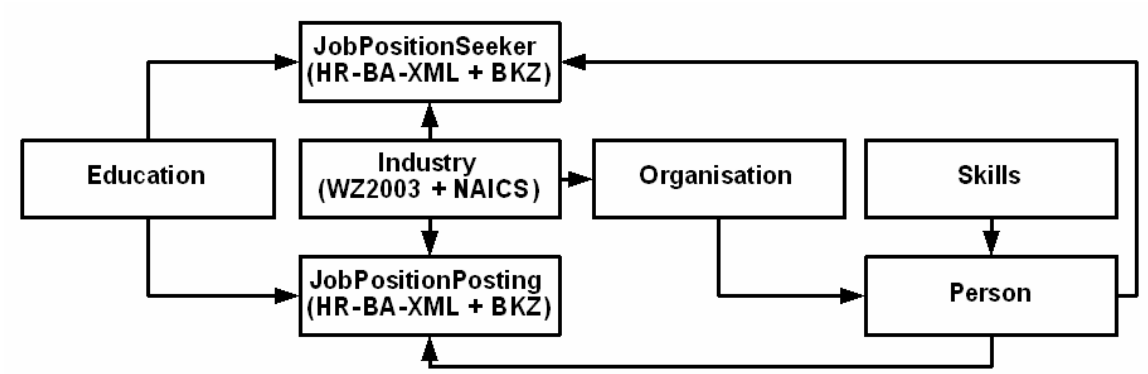

Figure 3: Sub-ontologies within the HR-ontology.

The skills sub-ontology defines concepts representing competencies, which are used to describe job requirements as well as applicant's skills. This ontology also provides means for specifying the competence level of a certain skill. We derived the skill sub-ontology from the KOWIEN ontology [Alan03]. Furthermore, we developed a person sub-ontology for describing personal information of job applicants and an Organization sub-ontology to specify the employer's company.

Our HR ontology is modelled using OWL [W3C04c] in order to allow accurate schema validation of incoming data. Descriptions of job postings and applicants' profiles are stored in RDF [W3C04a] using the vocabulary defined by the HR ontology.

\subsection{Data Integration Infrastructure}

Employers can use two different approaches for publishing annotated job postings depending on their existing software infrastructure. If they are using database backed standard software, they can export relational data directly into RDF using mapping tools like [Bize03, Bize04]. If they are not using any tools for managing open positions, they can annotate the HTML versions of their postings using annotation tools like [KaHe02]. The created RDF data is published using standard web servers like Apache or specialised query interfaces like the RDF NetAPI [MoSe03].

A job portal which is presenting the published data to job seekers will:

- use a directory service to locate information providers;

- use a crawler to collect data from the different providers;

- use a mapping engine to integrate data published using different vocabularies;

- provide semantic matching services to match applicant's profiles and job postings based on the knowledge included in the ontologies and the available market data; 
- render the data as HTML according to the user's preferences (level of detail, device, language, ....).

Following the above architecture, a portal will have to store huge amounts of collected RDF data and provide high performance access to this data for its users. An overview of specialised databases and query languages can be found in [Beck03].

Within our prototype we use D2R MAP [Bize03] for mapping relational data into RDF, the RDF NetAPI [MoSe03] and RAP [O1Bi04] for publishing data and the Jena 2 framework [Carr04] extended with a semantic matching engine as a foundation for the portal implementation.

\subsection{Semantic Matching}

Semantic Matching is a technique which combines annotations using controlled vocabularies with background knowledge about a certain application domain. In our scenario, the domain specific knowledge is represented in the form of various concept hierarchies (skills, occupation classification, industry sectors, etc.) and can be used to determine the semantic similarity between concepts [PoCa95]. This allows us to compare job descriptions and applicants' profiles based on their semantic similarity and not merely relying on the containment of keywords.

Our approach is based on ideas from [ZhZh02] and [BiSa02]. The similarity between two concepts $\mathrm{c}_{1}$ and $\mathrm{c}_{2}$ is determined by the distance $d_{c}\left(\mathrm{c}_{1}, \mathrm{c}_{2}\right)$ between them, which reflects their respective positions in the concept hierarchy. Concept similarity is defined as:

$$
\operatorname{sim}_{c}\left(c_{1}, c_{2}\right)=1-d_{c}\left(c_{1}, c_{2}\right)
$$

Every node in a concept hierarchy is assigned a milestone value [ZhZh02], which is calculated with the formula:

$$
\text { milestone }(n)=\frac{1 / 2}{k^{l(n)}}
$$

$k$ is a factor larger than 1 , indicating the rate at which the value decreases along the hierarchy. $l(n)$ is the depth of the node $n$ in hierarchy.

Since the distance between two given concepts in a hierarchy represents the path from one concept to the other one over the closest common parent ( $c c p)$, we calculate the distance as shown below:

$$
\begin{aligned}
& d_{c}\left(c_{1}, c_{2}\right)=d_{c}\left(c_{1}, c c p\right)+d_{c}\left(c_{2}, c c p\right) \\
& d_{c}(c, c c p)=\text { milestone }(c c p)-\text { milestone }(c)
\end{aligned}
$$


This model implies two assumptions: The semantic differences between upper level concepts are bigger than those between lower level concepts (in other words: two general concepts are less similar than two specialized ones) and that the distance between 'brothers' is greater than the distance between 'parent' and 'child'.

As an example, we calculate the distance between two concepts from our skill ontology: 'PropertyLaw' and 'CompanyLaw'. Figure 4 shows the corresponding ontology segment together with milestone values for ontology levels.

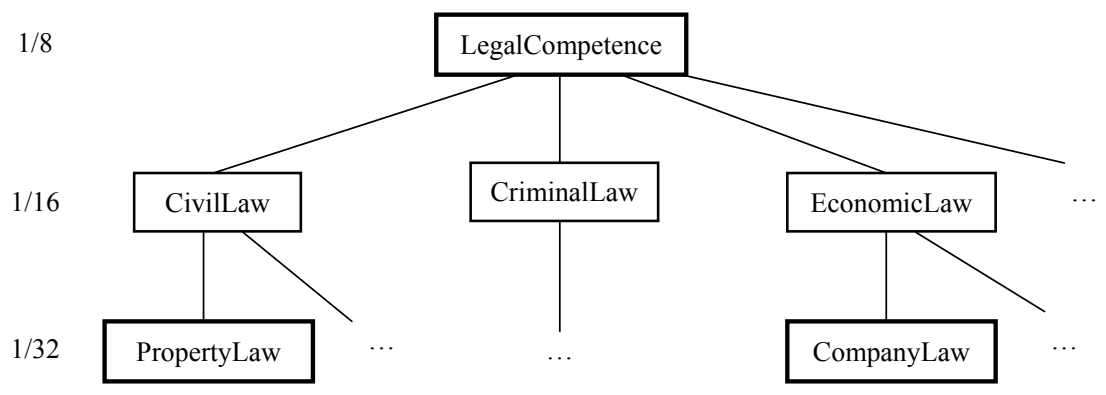

Figure 4: Skill ontology segment together with milestone values.

Since the closest common parent is 'LegalCompetence', the distance between the two concepts is calculated as follows:

$$
\begin{aligned}
d_{c}(\text { PropertyLaw, CompanyLaw })= & d_{c}(\text { PropertyLaw, LegalCompetence }) \\
& +d_{c}(\text { CompanyLaw, LegalCompetence }) \\
= & (1 / 8-1 / 32)+(1 / 8-1 / 32)=0,1875
\end{aligned}
$$

Consequently, the similarity between the two concepts is:

$\operatorname{sim}_{c}($ PropertyLaw, CompanyLaw $)=1-0,1875=0,8125$

We also provide means for specifying required competence levels $(c l)$ in job postings. Hence, our algorithm not only considers taxonomic similarity of concepts but also compares competence levels in order to find the best match. The competence level similarity is determined by the following formula:

$$
\operatorname{sim}_{p}\left(c l_{1}, c l_{2}\right)= \begin{cases}1-\alpha\left(c l_{1}-c l_{2}\right) & \mid c l_{1}-c l_{2} \geq 0 \\ 1 & \mid c l_{1}-c l_{2}<0\end{cases}
$$

where $0 \leq \alpha \leq 0,25$ is a factor indicating the rate at which the value of $\operatorname{sim}_{\mathrm{p}}$ decreases with increasing deviation between competence levels. For example, comparing an expert $(\mathrm{cl}=5)$ in property law with a novice $(\mathrm{cl}=1)$ in company law and setting $\alpha=0,2$ results in $\operatorname{sim}_{\mathrm{p}}=1-0,2(5-1)=0,2$. Multiplying this value with the concept similarity yields:

$\operatorname{sim}_{\mathrm{c}}\left(\right.$ PropertyLaw, CompanyLaw) ${ }^{*} \operatorname{sim}_{\mathrm{p}}($ expert, novice $)=0,8125 * 0,2=0,1625$. 
Our approach gives employers also the opportunity to specify the importance of different job requirements. The concept similarity is then justified by the indicated 'weight', i.e. the similarity between more important skills will have greater influence on the similarity between a job position posting and an applicant's profile.

Putting all together, the formula for calculating the similarity of a job position posting (jpp) and a job position seeker (jps) is:

$$
\begin{aligned}
& \operatorname{Sim}(j p p, j p s)=\sum_{i \in I} w\left(c_{j p p}^{i}\right) \cdot \max \left\{\operatorname { s i m } _ { c } ( c _ { j p p } ^ { i } , c _ { j p s } ^ { j } ) \cdot \operatorname { s i m } _ { p } \left(p\left(c_{j p p}^{i}\right), p\left(c_{j p s}^{j}\right)\right.\right. \\
& \mid j \in J\} \text { where } \sum_{i \in I} w\left(c_{j p p}^{i}\right)=1
\end{aligned}
$$

Each required skill from the job position posting $\left(c_{j p p}^{i}\right)$ is compared with each skill in an applicant's profile $\left(c_{j p s}^{i}\right)$. This includes the calculation of both concept and competence level similarities. The similarity values of the best matching pairs are multiplied by the corresponding weight and summed up yielding the final similarity.

\section{Economic Implications}

The possibility of higher market transparency raises new strategic questions for the market participants which will ultimately determine their willingness to participate: Is the scenario of economic interest for all participants? Is it possible to construct an economic win-win situation which would motivate all parties to participate?

This section will briefly discuss the possible implications of the realization of our scenario on the business models of the participants.

Online job portals are information intermediaries between job seekers and employers. The business model of charging publication fees, which portals are using today, prevents employers from publishing their postings on a larger number of job portals. This leads to a division of the online labour market into information islands and makes it close to impossible for a job seeker to get an overview of all relevant open positions.

Using Semantic Web technology would change information flows. An open position published on an employer's website can be used by all job portals. The formerly controlled communication links change to an open n:m communication situation. 
This would allow job seekers to use one single portal of their choice to access all information available in the market. Employers would save publication fees by publishing open positions only on their own website. The additional costs of annotating job postings are likely to be overcompensated by savings in publication fees, which usually amount to several hundred euros.

The crucial question is the business model of job portals within our recruitment scenario. How will portals finance their services, if they can not rely on publication fees? There are several options. Portals could be financed by:

- service charges paid by job seekers for access to market data and additional services like semantic matching;

- consortiums of employers, which could form in order to reach more potential applicants and save publication fees;

- public authorities, who have an interest in smoothing information flows in the labour market in order to cut unemployment.

Another question is the financing of maintaining the controlled vocabularies used in the market. This could also be a task for a public agency - like the German Federal Employment Office (BA) - as a market maker or could be accomplished by a non-profit consortium of market participants, similar to the process already taking place within the HR-XML Consortium.

\section{Conclusion and Outlook}

We presented a scenario for supporting recruitment processes with Semantic Web technologies. We described the online recruitment process and analysed the stateof-the-art technologies in this domain. We suggested improvements for several steps by enhancing job postings and job applications with annotations using controlled vocabularies. We described our prototypical implementation of the scenario. The core components of the prototype are a human resource ontology derived from existing standards, a data publishing and integration infrastructure and a retrieval engine using semantic match-making.

The creation of the ontology and the prototypical implementation of the software infrastructure showed that our scenario is technologically realizable. The analysis of the interest of the participants and the potential economic impacts showed that the main actors in the employment market - employers and job seekers - would both benefit from the realization of the scenario. Job seekers would benefit from the increased market transparency. Employers would benefit from reaching more potential applicants, saving money on publication fees and from cutting costs by automating the preselection of applications. 
We will concentrate our future work on the completion of the prototype, the creation of a larger real-world dataset of annotated job postings for testing the scalability of the infrastructure, and on improving the semantic matching algorithm.

\section{Acknowledgements}

This work is part of the Knowledge Nets project whose aim is to research the impact of the Semantic Web technologies on electronic markets [ToBi04]. KnowledgeNets belongs to the InterVal - Berlin Research Centre for the Internet Economy [InterVal04] and is funded by the German Ministry of Research BMBF.

\section{References}

[Alan03] Alan, Y.: Konstruktion der KOWIEN-Ontologie. Projektbericht 2/2003, Institut für Produktion und Industrielles Informationsmanagement, Universität Duisburg-Essen, www.kowien.uni-essen.de/publikationen/konstruktion.pdf, 2003, Download 2004-0625.

[Beck03] Beckett, D.: Scalability and Storage: Survey of Free Software / Open Source RDF storage systems. http://www.w3.org/2001/sw/Europe/reports/rdf_scalable_storage _report/, 2003, Download 2004-06-20.

[BeHe01] Berners-Lee, T.; Hendler, J.; Lassila, O.: The Semantic Web. A new form of Web content that is meaningful to computers will unleash a revolution of new possibilities. http://www.scientificamerican.com/, 2001, Download 2004-06-25.

[BiSa02] Billig, A.; Sandkuhl, K.: Match-Making based on Semantic Nets: The XMLbased approach of BaSeWeb. In: Tolksdorf, R., Eckstein, R. (Edt.): Proceedings of the 1st workshop on XML-Technologien für das Semantic Web, 2002, pp. 39-51.

[Bize03] Bizer, C.: D2R MAP - A Database to RDF Mapping Language. In: Proceedings of the $12^{\text {th }}$ International World Wide Web Conference, 2003.

[Bize04] Bizer, C.; Seaborne, A.: D2RQ - Treating Non-RDF Databases as Virtual RDF Graphs. In: Proceedings of the $3^{\text {rd }}$ International Semantic Web Conference, 2004.

[Carr04] Carroll, J.; et al.: Jena: Implementing the Semantic Web Recommendations. In: Proceedings of the $13^{\text {th }}$ International Wourld Wide Web Conference, 2004.

[Cross03] Crosswater Systems Ltd.: Der Virtuelle Arbeitsmarkt der Bundesanstalt für Arbeit. Anspruch und Wirklichkeit - das Millionengrab. http://www.crosswatersystems.com/dossier_03_vam_fakten_v100.pdf, 2003, Download 2004-06-25.

[HeBe02] Hendler, J.; Berners-Lee, T.; Miller, E.: Integrating Applications on the Semantic Web. Journal of the Institute of Electronic Engineers of Japan 122, 2002, pp. 676-680. 
[InterVal04] Günther, O.; et al.: InterVal-Berlin Research Center for the Internet Economy. http://interval.hu-berlin.de/, 2004, Download 2004-06-20.

[KaHe02] Kalyanpur, A.; Parsia, B.; Hendler, J.; Golbeck, J.: SMORE - Semantic Markup, Ontology, and RDF Editor. http://www.mindswap.org/papers/SMORE.pdf, 2003, Downlaod 2004-06-25.

[MoSe03] Moore, G.; Seaborne, A.: RDF Net API, W3C Member Submission. http://www.w3.org/Submission/2003/SUBM-rdf-netapi-20031002/, 2003, Download 2004-06-20.

[OlBi04] Oldakowski, R.; Bizer, C.: RAP: RDF API for PHP. http://www.wiwiss.fuberlin.de/suhl/radek/pub/RAP-oldakowski.pdf, 2004. Download 2004-09-21.

[PoCa95] Poole, J.; Campbell, J.A.: A Novel Algorithm for Matching Conceptual and Related Graphs. In: Ellis, G., et al. (Edt.): Conceptual Structures: Applications, Implementation and Theory. Volume 954 of LNAI, Springer Verlag, 1995, pp. 293-307.

[QuKa04] Quan, D.; Karger, D.: How to Make a Semantic Web Browser. In: Proceedings of the $13^{\text {th }}$ International Wourld Wide Web Conference, 2004.

[Schr04] Schraefel, M.; et al.: CS AKTive Space: Representing Computer Science in the Semantic Web. In: Proceedings of the $13^{\text {th }}$ International Wourld Wide Web Conference, 2004.

[ToBi04] Tolksdorf, R.; Bizer, C.; Eckstein, R.; Heese, R.: Trustable B2C Markets on the Semantic Web, Journal on Computer Systems Science \& Engineering, 2004. To appear.

[W3C04a] W3C: RDF/XML Syntax Specification (Revised). http://www.w3.org/TR/rdfsyntax-grammar/, 2004, Download 2004-06-25.

[W3C04b] W3C: RDF Vocabulary Description Language 1.0: RDF Schema. http://www.w3.org/TR/rdf-schema/, 2004, Download 2004-06-25.

[W3C04c] W3C: OWL Web Ontology Language-Reference. http://www.w3.org/TR/owlref/, 2004, Download 2004-06-25.

[WiMo03] Institut für Wirtschaftsinformatik der Johann Wolfgang Goethe-Universität Frankfurt am Main; Monster Deutschland and TMP Worldwide: Recruiting Trends 2004. 2. Fachsymposium für Personalverantwortliche, 2003.

[ZhZh02] Zhong, J.; Zhu, H., Li, J.; Yu, Y.: Conceptual Graph Matching for Semantic Search. In: Proceedings of the 2002 International Conference on Computational Science, 2002. 
